\title{
Smart Shoe for Tracking and Monitoring of Army Soldiers
}

\author{
S Jessica Pauline ${ }^{\mathrm{a}, 1}$, Bharkavi A $\mathrm{G}^{\mathrm{b}}$, Annlet Jesgna ${ }^{\mathrm{b}}$, Maheswari $\mathrm{S}^{\mathrm{b}}$ \\ ${ }^{a, 1}$ UG Scholar, Dept of ECE, Panimalar Engineering College, Chennai, India \\ ${ }^{b}$ Professor, Dept of ECE, Panimalar Engineering College, Chennai, India
}

\begin{abstract}
Presently in India, all sections of the defence system like army, navy and air force are facing an issue of tracking the soldier. At present, to know the status and location of each soldier, soldiers are communicated over radio line. But these methods are not effectivet for tracking. At the time of war, we always hear the problem of missing of soldiers in the newspaper. On focusing this problem, we have decided to develop a system that will automatically trace the location of each soldier. This paper utilizes long distance RF communication modules such as LoRa modules which provide long distance communication in terms of kilometres. As the military personnel moves around the city, the location of the military personnel is been transmitted to a nearby LoRa which is then transmitted to the mobile app developed independently for tracking the military personnel. Thus this paper will help in effectively tracking the military personnel.
\end{abstract}

Keywords: LoRa, RF communication, Tracking, Military personnel.

\section{Introduction}

In the existing system, in order to examine the execution of LoRa radios for industrial modernization both plain and energy gathering industrial territories are taken into account. The cost commutation inspection betwixt battery substitution and injury fine beside contrast sensing intervals which explains a direct rise in entire cost is the conclusion for these considerations taken into account. The work proposed a version to gauge the energy consumption, evaluating the battery span of LoRaWAN observing instruments. No practical communication in terms application is been implemented using the LoRa modules. This project utilizes long distance RF communication modules such as LoRa modules which provide long distance communication in terms of kilometres. The hardware component includes micro-controller, GPS, LoRa modules, etc. The hardware module is fixed to the shoes of the military personnel. As the military personnel moves around the city, th elocation of the military personnel is been The hardware component includes micro- controller, GPS, LoRa modules, etc. The hardware module is fixed to the shoes of the military personnel. As the military personnel moves around the city, the location of the military personnel is been transmitted toa near LoRa which is then transmitted to mobile app developed independently for tracking the military personnel. Thus this paper will help in effectively tracking the military personnel. Cheap and effective solution for tracking the military personnel.

\footnotetext{
${ }^{1}$ Bharkavi A G, UG Scholar, Dept of ECE, Panimalar Engineering College, Chennai, 


\section{Related works}

LoRa radio for industrial automation has been proposed by Hafiz Husnain Raza Sherazi et.al[1]. For the real time flows the assistance is given by LoRa. It describes a model to estimate the energy utilization, evaluating the battery period of LoRa WAN. RT-LoRa has been proposed by Luca Leonardi et.al[2], which offers assistance for real-time flows. The RT-LoRa gives some directions for the arrangement of an RT-LoRa network. To offer an individual electricity retail plan recommender system for domestic users, Fengji Luo et.al[3] has came up with service computing technique into the smart grid. The proposed personalized recommender system (PRS) is formed on the collaborative filtering (CF) technique. The smart meter first collects the user's energy utilization data, and then it extracts the key energy utilization quality of user and reserves into a user knowledge database (UKD), along with the information of their chosen electricity retail plans. To reduce the power density shortage of current energy storage systems (ESSs) in pure electric vehicles (PEVs or EVs), a hybrid ESS (HESS), Chunhua Zheng et.al[4] has proposed a system, which contains a battery and a supercapacitor, is taken into account. The simulation results exhibit that the proposed strategy conserves electricity compared to the rule-based strategy and the single ESS case for the three driving cycles examined. A LoRa wireless network classification for electricity metering has been analysed by Mauricio C.Tom 'e et.al[5], in which the technology along with a event-based metering strategy led to a great quality signal reconstruction [6-10].

\subsection{Requirements}

The hardware requirements include Power supply Unit, WiFi-module-ESP8266, AVR microcontroller, GPS module, LoRa Transmitter, LoRa Receiver. The software requirements include Visual Studio, CV AVR/ Arduino, Extreme burner, Android Studio working description

\subsection{Block diagram}

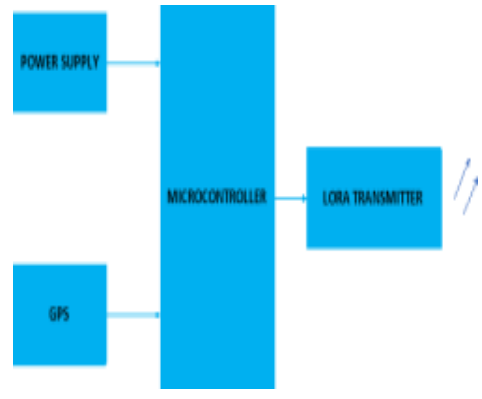

Figure 1. Transmitter Module

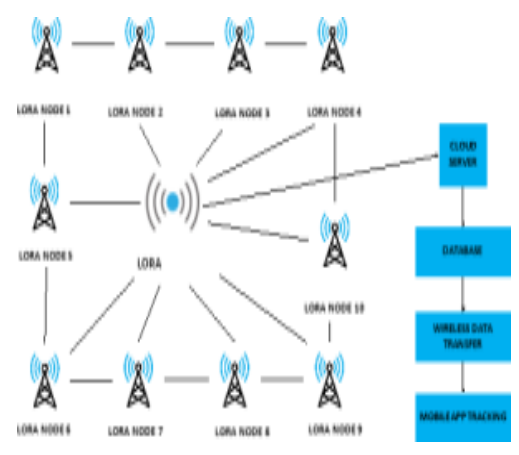

Figure 2. Receiver Module 


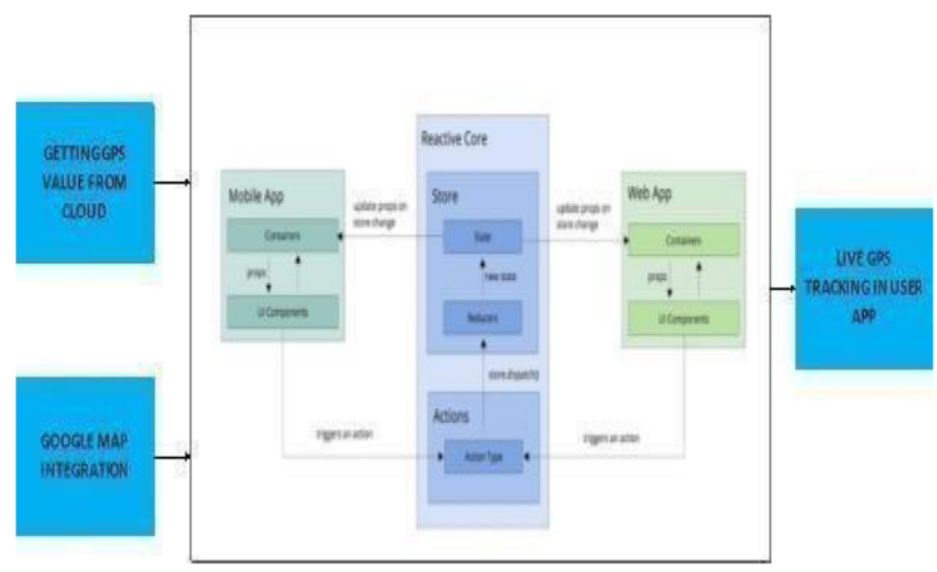

Figure 3. Internal mobile development architecture

\subsection{Working}

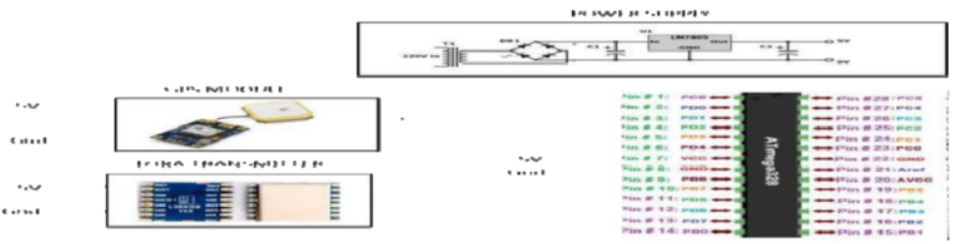

Figure 4. Transmitter circuit 1

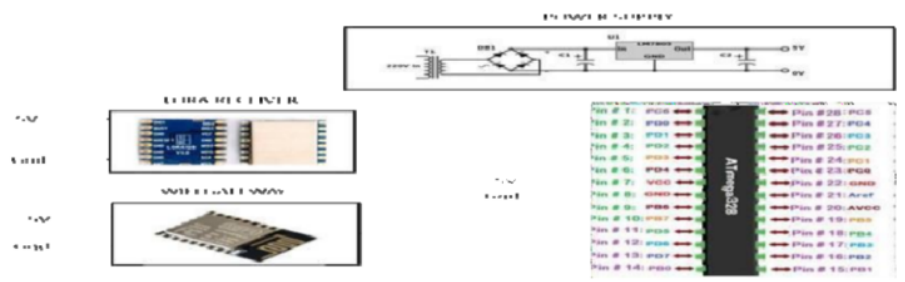

Figure 5. Receiver circuit 1

To start with the first step is to initiate the slave and master unit and then it initiates the LoRa RF module. LoRa technology offers long range, low power and secure data transmission for M2M and IoT applications. LoRa WAN defines the communication protocol and system architecture for the network by utilizing the unlicensed spectrum in the ISM bands and the long range communication which links remote sensors and gateways which are created by the LoRa physical layer. This protocol assists in the rapid setup of public or private IoT networks anywhere using hardware and software. Then it initiates the GPS module and then track the GPS location of the military people 
and transmit the obtained data to the nearby slave modules. The Fig. 4 shows the transmitter circuit consisting of GPS module, LoRa transmitter, power supply unit and ATmega microcontroller. Then it transmits data to master module and then it sends the data to the cloud and finally it receives the data and integrate into GPS tracking in mobile application. The Fig. 5 represents the receiver circuit. It consists of LoRa receiver, WiFi gateway, power supply unit and ATmega microcontroller. In this concept react native is used for mobile application development. React native is a structure which constructs an order of UI components to construct the Java script code. It contains of a group of modules for iOS and Android platforms in order to build a mobile application. React native is a practical solution for constructing good quality apps in a little time period with the exact performance and user-experience standards that native apps provide. React native apps are composed into natively written code, that allows React Native to function on both operating systems and also have similar properties on both platforms without any lag. Finally it tracks the location in the map and it intimates on connection loss

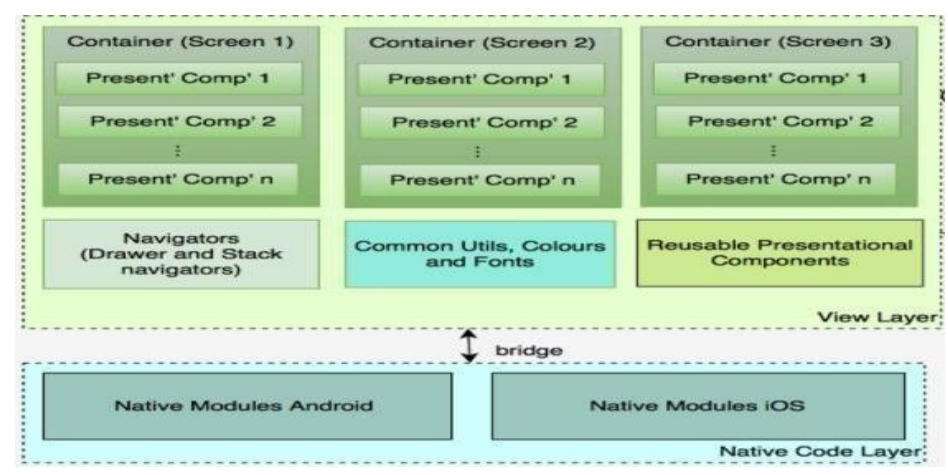

Figure 6. Mobile App developement 1

\section{Result and Discussion}

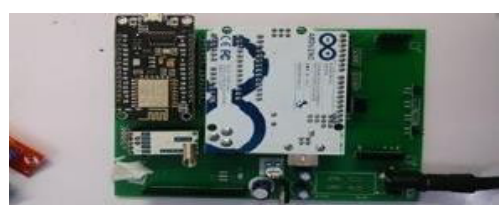

Figure 7. Transmitter Hardware Setup

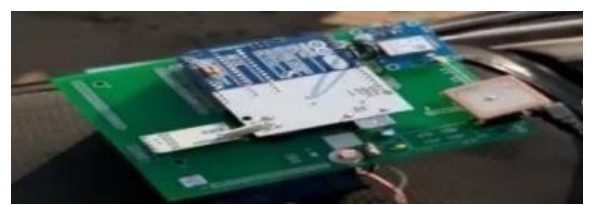

Figure 8. Receiver Hardware Setup 


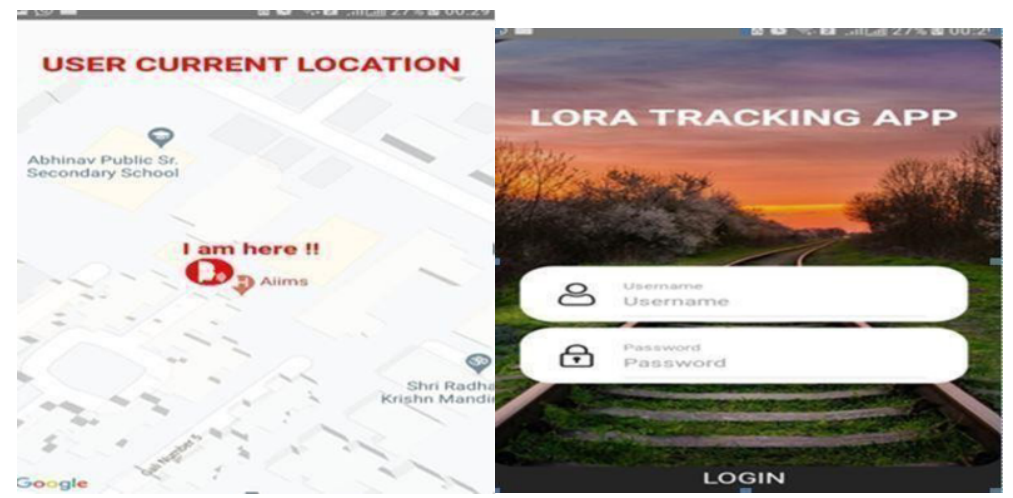

Figure 9. User location

Figure 10. LoRa tracking app

The Fig. 7 shows the transmitter hardware setup, which transmits the information to the LoRa receiver. Fig. 8 shows the receiver hardware setup. Fig. 9 shows the exact location of the user and Fig. 10 shows the LoRa tracking application which can be accessed using username and password. From Fig. 9 the exact location can be determined and in case of anyemergency the soldier can be saved in an efficient way.

\section{Conclusion}

Thus this paper suggests a strong idea for tracking the military personnel in an efficient way. The applications of this paper include asset tracking in airports and in commercial spaces. In the coming future, the application of the LoRa technology in the safety field can be reviewed and it can promote for advance in the travel industry with more accuracy. In this field there are more chances to develop or convert this idea in many ways. Thus, this concept has an efficient scope in coming future where this idea can be converted to computerized production in a cheap way.

\section{Reference}

[1] Energy-efficient LoRaWAN for industry 4.0 Applications, Hafiz Husnain Raza Sherazi, Member, IEEE, Luigi Alfredo Grieco, Senior Member, IEEE, Muhammad Ali Imran, Senior Member, IEEE, and Gennero Boggio, Senior Member, IEEE, [2020, Vol No:1551-3203]

[2] RT-LoRa: A medium Access Strategy to support Real-time flows over LoRa-based networks for industrial IoT applications, Luca Leonardi, Filipo Battaglia, Lucia Lo Bello, Senior Member, IEEE [2019, VolNo:2327-4662]

[3] Social Information Filtering Based Electricity Retail Plan Recommender System for smart Grid End User, Fenji Luo, Member, IEEE, Gianluca Ranzi, Member, IEEE, Xibin Wang, and Zhao Yang Dong, Fellow, IEEE [IEEE 2019]

[4] An Energy Management Strategy of Hybrid Energy Storage Systems for Electric Vehicle Applications, Chunhua Zheng, Member, IEEE, Weimen Li, Member, IEEE, and Quan Liang [2018, Vol No:2162237X]

[5] Long-range Low-power Wireless Networks and Sampling Strategies in Electricity Metering, MauricoC. Tom' e, IEEE Student Member, Pedro H.J. Nardelli, and Hirley Alves, IEEE Member [2018,VolNo:027 